\title{
Revisiting the quantum decoherence scenario as an explanation for the LSND anomaly
}

\author{
Pouya Bakhti, ${ }^{a}$ Yasaman Farzan $^{a}$ and Thomas Schwetz ${ }^{b}$ \\ ${ }^{a}$ Institute for research in fundamental sciences (IPM), \\ PO Box 19395-5531, Tehran, Iran \\ ${ }^{b}$ Oskar Klein Centre for Cosmoparticle Physics, \\ Department of Physics, Stockholm University, \\ SE-10691 Stockholm, Sweden \\ E-mail: pouya_bakhti@ipm.ac.ir, yasaman@theory.ipm.ac.ir, \\ schwetz@fysik.su.se
}

ABSTRACT: We propose an explanation for the LSND anomaly based on quantum decoherence, postulating an exponential behavior for the decoherence parameters as a function of the neutrino energy. Within this ansatz decoherence effects are suppressed for neutrino energies above $200 \mathrm{MeV}$ as well as around and below few $\mathrm{MeV}$, restricting deviations from standard three-flavour oscillations only to the LSND energy range of $20-50 \mathrm{MeV}$. The scenario is consistent with the global data on neutrino oscillations, alleviates the tension between LSND and KARMEN, and predicts a null-result for MiniBooNE. No sterile neutrinos are introduced, conflict with cosmology is avoided, and no tension between short-baseline appearance and disappearance data arises. The proposal can be tested at planned reactor experiments with baselines of around $50 \mathrm{~km}$, such as JUNO or RENO-50.

Keywords: Beyond Standard Model, Neutrino Physics

ARXIV EPRINT: 1503.05374 


\section{Contents}

1 Introduction 1

2 Quantum decoherence and the LSND anomaly 2

3 Analysis of short baseline and reactor neutrino data 4

3.1 Description of the used data and analysis details 4

3.2 Results of our fit 5

4 Predictions for future experiments and possible experimental tests 9

5 Conclusions

\section{Introduction}

The three neutrino mass and mixing scheme has been established as the standard paradigm to explain solar, atmospheric, long baseline and reactor experiment neutrino data. There are however some anomalies that cannot be explained within this standard paradigm. Arguably the most significant one is the LSND anomaly [1]. The canonical solution to the LSND anomaly is the four neutrino mixing scheme that includes a sterile neutrino with mass of order of $1 \mathrm{eV}$ and a small mixing with $\nu_{e}$ and $\nu_{\mu}$. This solution suffers from some drawbacks. Most importantly, within this scheme there is a tension between appearance and disappearance experiments, see [2-4] for recent analyses. Moreover, a sterile neutrino with mass and mixing parameters to solve the LSND anomaly is in tension with cosmology [5-7].

In view of these tensions, quantum decoherence has been suggested in the literature to explain LSND [8-10]. It is hypothesized that the evolution of quantum states receives a correction relative to the prediction of standard quantum mechanics. Such effects could arise in certain quantum gravity scenarios [11-13]. To explain the results of short baseline neutrino experiments, a phenomenological approach is taken to determine the form and magnitude of decoherence. Neutrino oscillation is a quantum interference effect over macroscopic distances, which can be sensitive to small corrections to quantum mechanics. The idea of involving quantum decoherence to explain the LSND anomaly was first proposed in $[8,9]$. As discussed in [10], within the framework proposed in $[8,9]$, the decoherence effects exceed the upper bound from the $\mathrm{NuTeV}$ experiment [14] in which neutrinos have an average energy of $75 \mathrm{GeV}$.

Considering this observation, the so-called soft decoherence scenario was suggested in [10] as a solution to the LSND anomaly. Within this scenario, the decoherence effects rapidly decrease with neutrino energy, avoiding the $\mathrm{NuTeV}$ bound and leaving neutrino 
oscillations in experiments with $\mathrm{GeV}$ scale neutrino energies unaffected. Furthermore, decoherence is restricted to the 1-3 sector, while the 1-2 sector is not modified. This explanation does not suffer from the appearance-disappearance tension of sterile neutrino models. On the other hand, with the power law energy dependence that was assumed in [10], reactor neutrinos undergo quantum decoherence after propagating distances of few $\mathrm{cm}$. This implies that no oscillation due to $\Delta m_{31}^{2}$ would be possible along the distance between near and far detectors of reactor experiments. For this reason, the soft decoherence scenario of [10] is now excluded by the results of the Daya Bay $[15,16]$ and RENO $[17,18]$ experiments. ${ }^{1}$ In the present paper, we revisit the decoherence scenario by modifying the power law assumed in [10] to an exponential energy dependence of the decoherence parameter, leading to an explanation of LSND consistent with all existing data.

The outline of the paper is as follows. In section 2 we review the decoherence scenario and introduce the ansatz for the exponential energy dependence of the decoherence coefficients. Section 3 contains the numerical results of our analysis of the relevant oscillation data, showing that the scenario can explain LSND without being in conflict with other data. In section 4 we discuss further implications of the scenario and predictions for future experiments. In particular, we show that planned intermediate baseline $(50 \mathrm{~km})$ reactor experiments can provide a crucial test of the framework. We conclude in section 5 .

\section{Quantum decoherence and the LSND anomaly}

In the quantum decoherence framework, the evolution of the density matrix for neutrinos can be described as

$$
\frac{d \rho}{d t}=-i[H, \rho]-\mathcal{D}[\rho]
$$

where $H$ is the Hamiltonian and $\mathcal{D}[\rho]$ parameterizes the decoherence effects. Maintaining complete positivity leads to the Lindblad form for $\mathcal{D}[\rho][20,21]$

$$
\mathcal{D}[\rho]=\sum_{m}\left[\left\{\rho, D_{m} D_{m}^{\dagger}\right\}-2 D_{m} \rho D_{m}^{\dagger}\right]
$$

where $D_{m}$ are general complex matrices. Unitarity then requires $D_{m}$ to be Hermitian. If we further impose conservation of average energy, we find $\left[H, D_{m}\right]=0$. As a result, in the neutrino mass basis, $D_{m}$ and $H$ can be simultaneously diagonalized

$$
H=\operatorname{Diag}\left[h_{1}, h_{2}, h_{3}\right], \quad D_{m}=\operatorname{Diag}\left[d_{m, 1}, d_{m, 2}, d_{m, 3}\right],
$$

where $h_{i}=\left(p^{2}+m_{i}^{2}\right)^{1 / 2}$ (adopting the equal momentum approximation for the mass states), and $d_{m, i}$ are unknown energy dependent real quantities with dimension of $[\text { mass }]^{1 / 2}$. Solving eq. (2.1), we find

$$
\rho(t)=\left[\begin{array}{ccc}
\rho_{11}(0) & \rho_{12}(0) e^{-\left(\gamma_{12}-i \Delta_{12}\right) t} & \rho_{13}(0) e^{-\left(\gamma_{13}-i \Delta_{13}\right) t} \\
\rho_{21}(0) e^{-\left(\gamma_{21}-i \Delta_{21}\right) t} & \rho_{22}(0) & \rho_{23}(0) e^{-\left(\gamma_{23}-i \Delta_{23}\right) t} \\
\rho_{31}(0) e^{-\left(\gamma_{31}-i \Delta_{31}\right) t} & \rho_{32}(0) e^{-\left(\gamma_{32}-i \Delta_{32}\right) t} & \rho_{33}(0)
\end{array}\right]
$$

\footnotetext{
${ }^{1}$ Note that the DoubleChooz experiment [19] does not (yet) exclude this scenario, since no data on near-far comparison is available to date.
} 
in which

$$
\gamma_{i j} \equiv \sum_{m}\left(d_{m, i}-d_{m, j}\right)^{2} \text { and } \Delta_{j i} \equiv h_{j}-h_{i} \approx \frac{\Delta m_{j i}^{2}}{2 E_{\nu}} .
$$

Obviously, $\gamma_{i j}=\gamma_{j i}$ and $\Delta_{i j}=-\Delta_{j i}$. This means $\gamma_{i j}$ is symmetric under flipping $i \leftrightarrow j$. In the following, we assume that only one term contributes in the sum and we drop the index $m$. The flavor conversion probability can be written as

$$
P_{\alpha \beta}=\left\langle\nu_{\beta}\left|\rho^{(\alpha)}(t)\right| \nu_{\beta}\right\rangle=\sum_{i j} U_{\beta i}^{*} U_{\beta j} \rho_{i j}^{(\alpha)}(t)
$$

where $U_{\alpha i}$ are the elements of the PMNS matrix [22, 23]. The density matrix $\rho_{i j}^{(\alpha)}(t)$ is given by eq. (2.4) and $\rho_{i j}(0)=\rho_{i j}^{(\alpha)}(0)=U_{\alpha i} U_{\alpha j}^{*}$. The flavor conversion probability for antineutrinos, $P_{\bar{\alpha} \bar{\beta}}$, will be given by a similar formula, replacing $U$ with $U^{*}$.

In the soft decoherence scenario of [10], a power law energy dependence of the decoherence coefficients has been assumed, $d_{i} \propto E^{-r}(r \geq 2)$, suppressing decoherence effects for $E \gtrsim 100 \mathrm{MeV}$. However, as mentioned in the introduction, this leads to strong decoherence effects at low energies and is by now excluded by Daya Bay and RENO results. In this work, we therefore propose a modified energy dependence of the decoherence parameters and we conjecture an exponential dependence on energy for $d_{i}$ as follows:

$$
d_{i}=\sqrt{\gamma_{0}} \exp \left[-\left(\frac{E}{E_{i}}\right)^{n}\right]
$$

where $\gamma_{0}$ is a constant parameter with dimension of mass, universal for all mass eigenstates. $E_{i}$ are also constant parameters with dimension of mass but can in principle take different values for different mass eigenstates. The power $n$ can take any arbitrary number. In line with the idea of soft decoherence, we take a value for $n$ and $E_{i}$ for which at energies $\gtrsim$ few $\times 100 \mathrm{MeV}$, the decoherence parameters become suppressed rapidly enough not to have any effects at experiments such as MINOS [24], T2K [25-27], atmospheric neutrinos [28, 29] and etc. In the same way this predicts null-results for short-baseline experiments with $E \gtrsim 200 \mathrm{MeV}$ such as MiniBooNE [30-32], CDHS [33], NOMAD [34], NuTeV [14] and etc. We found that with $E_{i}<100 \mathrm{MeV}$ and $n \geq 2$, this requirement is fulfilled. Unless it is stated otherwise, we take $n=2$ for definiteness throughout this paper.

To avoid constraints from the long-baseline KamLAND reactor experiment [35], we restrict the scenario to $d_{1} \approx d_{2}$ or equivalently to $\gamma_{12} \approx 0$ [10]. In the limit $\left|E_{1}-E_{2}\right| \ll E_{1}$ with taking $n=2$ and $E \lesssim E_{1}$, we find $\gamma_{12} \simeq 4 \gamma_{0} \exp \left(-2 E^{2} / E_{1}^{2}\right) E^{4}\left(E_{2}-E_{1}\right)^{2} / E_{1}^{6}$. To avoid bounds from KamLAND, $\gamma_{12}$ should be much smaller than $\sim(200 \mathrm{~km})^{-1}$ at $E \sim$ few $\mathrm{MeV}$ which for $E_{1} \gg \mathrm{MeV}$ means $\left|E_{2}-E_{1}\right| / E_{1} \ll\left(800 \mathrm{~km} \gamma_{0}\right)^{-1 / 2}\left[E_{1} /(\text { few MeV })\right]^{2}$. At first sight, it seems that from solar neutrino data, we can obtain strong bounds on $\gamma_{12}$, too. However, for long baselines, the interference effects are averaged out and as a result the sensitivity to $\gamma_{i j}$ is lost. This happens for solar neutrinos even before reaching the resonance region inside the Sun. From a theoretical point of view, it may be natural to assume that $d_{i}$ are functions of mass: $d_{i}=f\left(m_{i}\right)$. From $m_{1} \simeq m_{2} \neq m_{3}$, we then expect $d_{1} \simeq d_{2} \neq d_{3}$. 
In the rest of this paper, we shall take

$$
\gamma_{12}=0 \text { and } \gamma \equiv \gamma_{13}=\gamma_{32}=\gamma_{0}\left(\exp \left[-\left(\frac{E}{E_{3}}\right)^{n}\right]-\exp \left[-\left(\frac{E}{E_{1}}\right)^{n}\right]\right)^{2}
$$

with $n=2$. Notice that the combination in the parenthesis is less than or equal to 1 and hence, $\gamma \leq \gamma_{0}$. For $E \gg E_{1}, E_{3}$, we have $d_{1}, d_{3} \rightarrow 0$ and $\gamma$ will therefore exponentially converge to zero. For $E \ll E_{1}, E_{3}, \gamma$ will also be small and suppressed by $\left[E^{n}\left(E_{1}^{-n}-\right.\right.$ $\left.\left.E_{3}^{-n}\right)\right]^{2}$. Only for $E \sim E_{1}, E_{3}$, the value of $\gamma$ can be sizable and decoherence effects can be significant. Note that the suppression of decoherence at low energies works only for a universal coefficient $\gamma_{0}$. Hence, the assumption that $\gamma_{0}$ is independent of the neutrino mass is crucial for our scenario.

For $\Delta_{21} L \ll 1$, we can write

$$
\begin{aligned}
P_{\bar{\mu} \bar{e}}(\gamma, L)=P_{\mu e}(\gamma, L) & =P_{e \mu}(\gamma, L) \simeq 2\left|U_{\mu 3}\right|^{2}\left|U_{e 3}\right|^{2}\left[1-e^{-\gamma L} \cos \left(\Delta_{31} L\right)\right] \\
P_{\bar{e} \bar{e}}(\gamma, L)=P_{e e}(\gamma, L) & \simeq 1-2\left|U_{e 3}\right|^{2}\left(1-\left|U_{e 3}\right|^{2}\right)\left[1-e^{-\gamma L} \cos \left(\Delta_{31} L\right)\right], \\
P_{\bar{\mu} \bar{\mu}}(\gamma, L)=P_{\mu \mu}(\gamma, L) & \simeq 1-2\left|U_{\mu 3}\right|^{2}\left(1-\left|U_{\mu 3}\right|^{2}\right)\left[1-e^{-\gamma L} \cos \left(\Delta_{31} L\right)\right] .
\end{aligned}
$$

For $\gamma L \rightarrow 0$ the quantum decoherence is turned off and the flavor conversion probability becomes equal to that in the standard three neutrino oscillation scenario.

\section{Analysis of short baseline and reactor neutrino data}

In this section, we present the results from a numerical analysis of relevant data and determine the allowed range of parameters which can account for the LSND anomaly without being in conflict with any other experimental results.

\subsection{Description of the used data and analysis details}

In our analysis, we focus on the LSND electron antineutrino excess events in the energy range from $20 \mathrm{MeV}$ to $60 \mathrm{MeV}$ [1]. We extract the data points as well as the background from figure 24 of [1]. The data sample shown in that figure was obtained by applying the analysis cut $R_{\gamma}>10$, see section VII-C of [1] for the definition of the $R_{\gamma}$ variable. To predict the number of events in each bin within the decoherence scenario, we normalize the total number of events for $P\left(\bar{\nu}_{\mu} \rightarrow \bar{\nu}_{e}\right)=1$ to 33300 as indicated in table VIII of [1], multiplied by 0.39 which is the efficiency of the $R_{\gamma}>10$ cut (see table IX of [1]). The $\chi^{2}$ is defined as the sum of squares of the difference between prediction (signal+background) and observed number of events per bin divided by the square of the uncertainty. The sum is over bins with $20 \mathrm{MeV}<E<60 \mathrm{MeV}$ (10 bins) and the uncertainties in each bin are obtained from the error bars on the data points in figure 24 of [1], which account for both systematic and statistical uncertainties. We have checked that our analysis reproduces the allowed region for standard oscillations obtained in [1] with good accuracy.

We also take into account the results of the KARMEN experiment [36], which observes 15 events in the energy range from $16 \mathrm{MeV}$ to $52 \mathrm{MeV}$ with a predicted background of $15.8 \pm 0.5$ events. Any explanation of the LSND anomaly has to address the null-result of 
KARMEN, taking into account the very similar experimental configuration, with the main difference being the somewhat shorter baseline of KARMEN. Again we perform a fit to the binned energy spectrum (9 bins) and we can reproduce the official results in terms of sterile neutrino oscillations to good accuracy. For short baseline experiments such as LSND and KARMEN, $\Delta_{31} L \ll 1$, so we can use eq. (2.9) to write the conversion probability for neutrinos and antineutrinos as follows:

$$
P_{\bar{\mu} \bar{e}}(\gamma, L)=P_{\mu e}(\gamma, L)=2\left|U_{\mu 3}\right|^{2}\left|U_{e 3}\right|^{2}\left(1-e^{-\gamma L}\right) \approx\left|U_{e 3}\right|^{2}\left(1-e^{-\gamma L}\right)
$$

As discussed before, the data from Daya Bay and RENO, being consistent with the standard three neutrino oscillation scheme, can put bounds on the decoherence parameters $E_{1}$ and $E_{3}$. To derive the bounds, we analyze the energy spectrum of the $\bar{\nu}_{e}$ flux at the near and far detectors of Daya Bay shown in figure 2 of [15]. We read the data points for near detectors (EH1 and EH2) and far detector (EH3) from the upper panel in pairs of panels shown in figure 2 of [15], 75 data points in total. We read the background for each detector from the inset panels in this figure. Finally, having extracted the data and background, to calculate the number of events per bin without oscillation (i.e., for $P\left(\bar{\nu}_{e} \rightarrow \bar{\nu}_{e}\right)=1$ ), we use the data points displayed in the lower panels [(data-background)/predictions] of figure 2 of [15]. To calculate the number of events within the decoherence scenario, we then multiply this number with the probability in eq. (2.10), averaged over cross section, flux, and energy resolution. To compute the $\chi^{2}$, we equate uncertainties for each bin to the root of number of events per bin (i.e., the statistical uncertainty). The overall flux normalization is taken to be a free parameter to be fixed by the combined near and far detector fit. The distances between the various reactor and detector sites of the Daya Bay experiment are taken from table 2 of [16].

We also include the spectrum of fifty thousand inverse beta-decay candidate events of the far detector of the RENO experiment [17] and compare it with the prediction. Data points are taken from the right panel of figure 4 of [18] (26 data points), where the background is already subtracted. To compute the prediction of the decoherence scenario for each bin we multiply the oscillation prediction shown in figure 4 of [18] by the averaged survival probability in eq. (2.10) and divide by the oscillation probability with $\sin ^{2} 2 \theta_{13}=$ 0.094 and $\left|\Delta m_{31}^{2}\right|=2.32 \times 10^{-3} \mathrm{eV}^{2}$ as stated in figure 4 of [18]. These mass and mixing parameters are the best fit values that ref. [18] derives by using a MC simulation to fit both near and far detector data. For values of $E_{1}$ and $E_{3}$ of interest for solving the LSND anomaly $\left(E_{1} \sim E_{3} \sim\right.$ few $\left.10 \mathrm{MeV}\right)$, decoherence at the near detector is negligible $(\gamma L \ll 1)$ and eq. (2.10) converges to the standard oscillation formula. Hence, using the far detector prediction based on the near detector data (as done for figure 4 of [18]) should be a good approximation. Notice, however, that including the RENO results does not much change the overall results for the decoherence fit, which is dominated by Daya Bay data.

\subsection{Results of our fit}

Remember that $P_{\bar{\mu} \bar{e}}$ at LSND should be of order few $\times 10^{-3}$ to account for the observed excess. For $\left|U_{e 3}\right|^{2} \simeq 0.02$, the value of $\gamma L$ for LSND should be of order of 0.1 to explain 

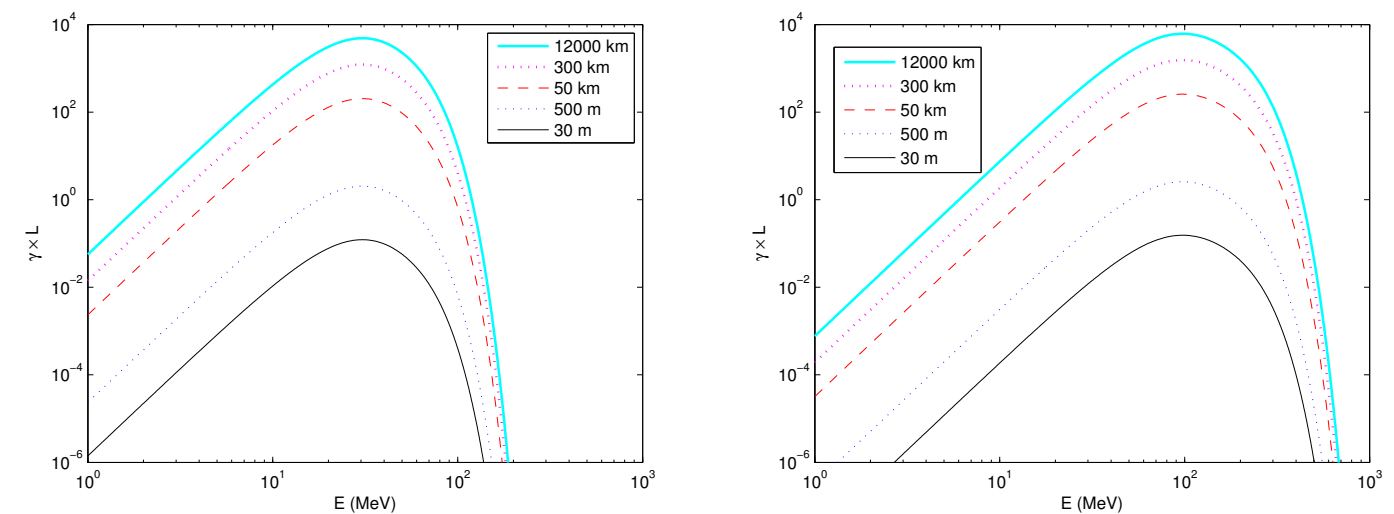

Figure 1. Dependence of $\gamma L$ on energy for different baselines corresponding approximately to LSND, MiniBooNE, medium baseline reactor, long baseline accelerator and atmospheric neutrino experiments. We have taken $n=2, \gamma_{0}=0.01 \mathrm{~m}^{-1}$ for both panels, and $E_{1}=E_{2}=20 \mathrm{MeV}$, $E_{3}=55 \mathrm{MeV}\left(E_{1}=E_{2}=60 \mathrm{MeV}, E_{3}=200 \mathrm{MeV}\right)$ for the left (right) panel.

the anomaly. From $\gamma<\gamma_{0}$ and $L=30 \mathrm{~m}$, we find that $\gamma_{0}$ has to be of order of $0.01 \mathrm{~m}^{-1}$ or larger. Larger values of $\gamma_{0}$ require $E_{1} \simeq E_{3}$ to cause partial cancelation, see eq. (2.8). To explain the LSND anomaly, we demand that $\gamma \sim \gamma_{0} \sim 0.01 \mathrm{~m}^{-1}$ at $E \sim 30 \mathrm{MeV}$ and to avoid the bounds from reactor experiment as well as from higher energy experiments, we require $\gamma \ll \gamma_{0}$ for both $E \gg 30 \mathrm{MeV}$ and $E \sim$ few $\mathrm{MeV}$. That means $E_{1}$ and $E_{3}$ should be of order of $10 \mathrm{MeV}$. Figure 1 shows $\gamma L$ versus energy taking typical values for decoherence parameters. The left panel of that figure corresponds to a parameter choice close to the best fit value of our model. As seen from figure 1 (left), at $\gamma_{0}=0.01 \mathrm{~m}^{-1}$, the effect of decoherence is negligible for energies above $200 \mathrm{MeV}$. Thus, the bounds from short-baseline experiments such as NOMAD, CDHS, or $\mathrm{NuTeV}$ are satisfied. In other words, like in the soft decoherence scenario, the tension between appearance and disappearance experiments plaguing the $3+1$ sterile oscillations is solved. Furthermore, the standard oscillation results for experiments with $\mathcal{O}(1 \mathrm{GeV})$ neutrinos such as MINOS, T2K, or atmospheric neutrinos are not affected. For $E_{i}>200 \mathrm{MeV}$ (see right panel of figure 1), decoherence effects can potentially show up in the low energy bins of $\mathrm{T} 2 \mathrm{~K}$ as well as in the sub- $\mathrm{GeV}$ atmospheric neutrino data.

Our main focus is on a range of parameters for which reactor and $\mathrm{T} 2 \mathrm{~K}$ experiments are unaffected. As a result, a reanalysis of Daya Bay and T2K will approximately yield the same value for $\theta_{13}$ as in the standard oscillation case. We fix the values of the standard neutrino parameters (including $\theta_{13}$ ) to the best fit value of the global analysis from [37]. We find that within this scenario with $n=2$, LSND data can be explained with a satisfactory p-value of $68 \%$ with three unknown parameters fitted to $\gamma_{0}=0.01 \mathrm{~m}^{-1}, E_{1}=E_{2}=18 \mathrm{MeV}$ and $E_{3}=63 \mathrm{MeV}$. The spectrum of events at LSND for these values is shown in figure 2. The figure demonstrates that data and prediction of the decoherence scenario are in good agreement. In the following analysis we will fix $\gamma_{0}$ to the LSND best fit value of $0.01 \mathrm{~m}^{-1}$.

Figure 3 shows the constraints from short baseline and reactor neutrino experiments on $E_{1}$ and $E_{3}$ at $90 \%$ C.L., fixing $n=2$ and $\gamma_{0}=0.01 \mathrm{~m}^{-1}$. As expected the bounds 


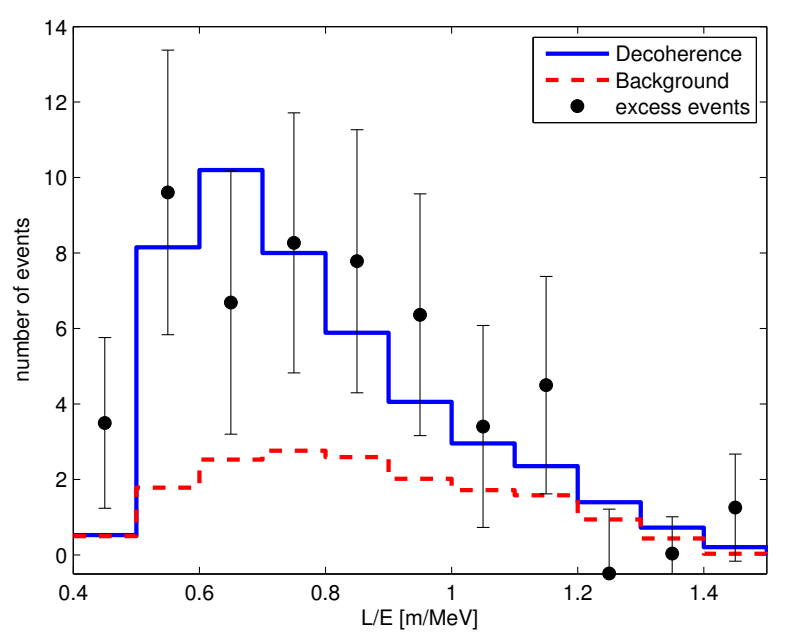

Figure 2. Decoherence prediction for LSND for $\gamma_{0}=0.01 \mathrm{~m}^{-1}, E_{1}=E_{2}=18 \mathrm{MeV}$ and $E_{3}=$ $63 \mathrm{MeV}$ compared with data.

are symmetric under $E_{1} \leftrightarrow E_{3}$. Due to the exponential dependence on the distance $L$, the difference in the baselines for LSND and KARMEN (30 m versus $18 \mathrm{~m}$ ) leads to a better consistency of the two results than in the case of oscillations. Figure 3 shows that at $90 \%$ C.L. KARMEN only marginally constrains the LSND allowed region, compare thin magenta (LSND) and dotted black (KARMEN) curves. For $E_{i} \lesssim 8 \mathrm{MeV}$, the bound from the reactor neutrino experiments becomes stringent and practically only the narrow region with $E_{1} \simeq E_{3}$ is allowed. But for $E_{1}, E_{3}>15 \mathrm{MeV}$, the bounds from reactor experiments are relaxed. The thick red curves in the plot show the globally allowed region. The best fit is marked by a cross in the plot and it is located at $E_{1}=20 \mathrm{MeV}$ and $E_{3}=56 \mathrm{MeV}$. We have clipped the figure at $E_{1}, E_{3}=200 \mathrm{MeV}$ because for larger values T2K and atmospheric neutrinos will also be affected, as visible from the right panel of figure 1.

Table 1 shows $\chi_{\min }^{2}$ per degrees of freedom and goodness of fit (GOF or p-value) for various short baseline and reactor neutrino experiments. Notice that the p-value for LSND given in the table (i.e., for the case that $\gamma_{0}$ is fixed and $E_{1}$ and $E_{3}$ are treated as free parameters) is better than the aforementioned p-value that we obtain when we treat $\gamma_{0}$ as a free parameter along with $E_{1}$ and $E_{3}$. This reflects the fact that for $\gamma_{0}>0.01 \mathrm{~m}^{-1}$, the minimum value of $\chi^{2}$ over the $E_{1}$ and $E_{3}$ plane does not change much by varying the value of $\gamma_{0}$. Let us comment on the somewhat large p-value of $93 \%$ for the reactor analysis. If Daya Bay and RENO are analyzed separately we find $\chi_{\min }^{2} / \mathrm{DOF}$ values of 55/72 and $23 / 24$, respectively. Hence, the too good fit comes from the Daya Bay analysis. This might be related to the accuracy of reading data from the plot. Note however, that our results are based on $\Delta \chi^{2}$ values, which are insensitive to the absolute value of the $\chi^{2}$. Furthermore, we can reproduce the standard $\theta_{13}$ result of Daya Bay with good accuracy.

Consistency of the combination of various experiments is quantified by the so-called Parameter Goodness of fit (PG) [38, 39] defined as

$$
\chi_{\mathrm{PG}}^{2}=\chi_{\mathrm{tot}, \min }^{2}-\sum_{i} \chi_{i, \min }^{2},
$$




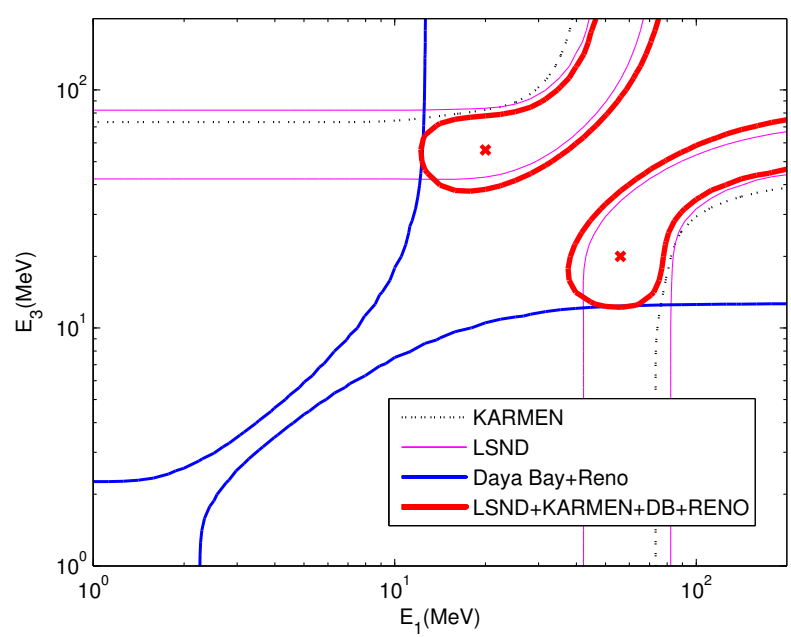

Figure 3. Constrains on the parameters $E_{1,3}$ from short baseline and reactor experiments at $90 \%$ C.L. taking $n=2$ and $\gamma_{0}=0.01 \mathrm{~m}^{-1}$. The region below and to the left of the dotted curves is allowed by KARMEN, the region between the dark-blue solid curves is allowed by Daya Bay and RENO, the thin magenta curves delimit the regions allowed by LSND. The regions consistent with all data are inside the thick red curves, with the cross indicating the best fit point.

\begin{tabular}{|lcccc|}
\hline Data & $\chi_{\min }^{2} / \mathrm{DOF}$ & $\mathrm{GOF}$ & $\chi_{\mathrm{PG}}^{2} / \mathrm{DOF}$ & $\mathrm{PG}$ \\
\hline LSND & $4.8 / 8$ & $77 \%$ & & \\
KARMEN & $7.0 / 7$ & $43 \%$ & & \\
Daya Bay and RENO & $78 / 98$ & $93 \%$ & & \\
LSND+KARMEN & $14 / 17$ & $66 \%$ & $2.3 / 2$ & $32 \%$ \\
LSND+KARMEN+Reactor & $93 / 118$ & $96 \%$ & $3.2 / 4$ & $52 \%$ \\
\hline
\end{tabular}

Table 1. $\chi_{\min }^{2} / \mathrm{DOF}$ and goodness of fit (GOF) for different combinations of short baseline and reactor neutrino data. The last two columns quantify the consistency of different experiments, see eq. (3.2) for the definition. $E_{1}=E_{2}$ and $E_{3}$ are taken as free parameters to fit the data and the rest are fixed to $\gamma_{0}=0.01 \mathrm{~m}^{-1}, n=2$ and $\sin ^{2} 2 \theta_{13}=0.085$.

where $\chi_{\text {tot,min }}^{2}$ is the global minimum, the sum over $i$ runs over the different experiments, and $\chi_{i, \min }^{2}$ are the minima of the experiments separately. As seen from the third and fourth columns of table 1, the KARMEN and LSND data are in good agreement with each other under the decoherence hypothesis, thanks to the exponential dependence of the transition probability on $L$. Furthermore, the short-baseline experiments LSND and KARMEN are also in very good agreement with the Daya Bay and RENO reactor experiments.

From figure 1, it is clear that decoherence effects are strongly suppressed for the MiniBooNE baseline of around $500 \mathrm{~m}$ and neutrino energies above $200 \mathrm{MeV}$. Thus, our scenario is consistent with a null-result in MiniBooNE, as observed in the energy range $E>475 \mathrm{MeV}[30,31]$. The low-energy event excess between 200 and $475 \mathrm{MeV}$ [40] is not explained since the transition probability is already highly suppressed in that regime. It 
is necessary to be consistent with $\mathrm{T} 2 \mathrm{~K}$ and atmospheric neutrino data, which requires us to restrict $E_{1,3}$ to values sufficiently low such that not to affect the standard oscillation behavior seen there. Hence the low-energy MiniBooNE excess has to find an alternative explanation.

\section{Predictions for future experiments and possible experimental tests}

First we mention that the so-called reactor [41-43] and Gallium [44] anomalies cannot be explained in the decoherence framework proposed here. At reactor energies and below, the decoherence effects are suppressed so we predict neither a reduced reactor neutrino flux at short baselines, nor a reduced neutrino rate in source experiments at Gallium detectors. Those anomalies (which are at the level of $3 \sigma$ ) should find another explanation in the scenario discussed here. Planned experiments at reactors with very short baselines as well as radioactive source experiments should lead to null-results.

Let us now comment on future accelerator-based experiments. Long-baseline oscillation experiments such as NOvA [45] or LBNF [46, 47] use neutrino beams with $E_{\nu} \gtrsim 1 \mathrm{GeV}$. As clear from figure 1 we predict no decoherence effects at those energies and hence such experiments should obtain results consistent with standard three-flavour oscillations. The nuSTORM short baseline neutrino experiment with an average energy of $3 \mathrm{GeV}$ and a baseline of $2 \mathrm{~km}$ is proposed to test the $3+1$ oscillation hypothesis [48]. From figure 1, we observe again that the decoherence effects for this setup are too small so we predict a null signal for such an experiment. If nuSTORM finds no signal for appearance, the $3+1$ solution will be ruled out but the decoherence solution will still survive, while the observation of an appearance signal at nuSTORM would exclude the decoherence solution proposed here. The situation is similar also for other short baseline neutrino experiment proposals with neutrino energies $\gtrsim$ few $\times 100 \mathrm{MeV}$, see e.g., [49, 50]. For the ESS superbeam [51, 52], with a peak energy of $E \simeq 200 \mathrm{MeV}$ some decoherence effects may start to show up if the $E_{1,3}$ parameters are not too small. We do predict an appearance signal in LSND-like short baseline experiments with energies around $30 \mathrm{MeV}$; see e.g., [53].

A crucial test of our scenario might be possible with reactor experiments with baselines of around $50 \mathrm{~km}$, such as the JUNO [54, 55] and RENO-50 [18] projects. Using the formalism of section 2 we obtain for the three-flavour $\bar{\nu}_{e}$ survival probability

$$
\begin{aligned}
P_{\bar{e} \bar{e}}= & 1-\sin ^{2} 2 \theta_{12} \sin ^{2} \frac{\Delta_{21} L}{2}-\frac{1}{2} \sin ^{2} 2 \theta_{13} \\
& +\frac{1}{2} \sin ^{2} 2 \theta_{13} e^{-\gamma L}\left[\cos ^{2} \theta_{12} \cos \left(\Delta_{31} L\right)+\sin ^{2} \theta_{12} \cos \left(\Delta_{32} L\right)\right] .
\end{aligned}
$$

The main goal of those experiments is to observe the small "wiggles" in the energy spectrum induced by the term in the second line of eq. (4.1). We see that for $\gamma L \gtrsim 1$ those features will be suppressed due to decoherence. Figure 1 (left) shows that for baselines of $50 \mathrm{~km}$, $\gamma L$ becomes of order one for $E_{\nu} \gtrsim 4 \mathrm{MeV}$, and hence, the fast oscillations in the survival probability may become suppressed.

We perform a numerical study of this effect by simulating the JUNO experiment, using information from [55]. We normalize the number of events such that for the default 

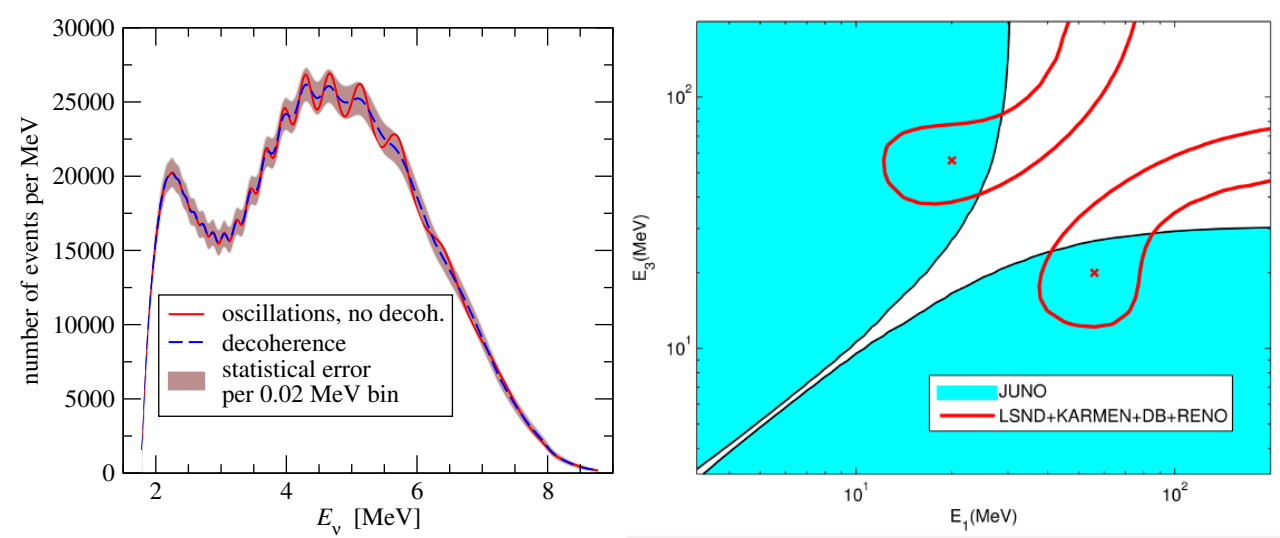

Figure 4. Left: event spectrum at JUNO for an exposure of $4320 \mathrm{kt} \mathrm{GW}$ yr. The red solid curve corresponds to standard oscillations with $\Delta m_{31}^{2}>0$, whereas the blue dashed curve shows the spectrum with decoherence parameters $E_{1}=E_{2}=20 \mathrm{MeV}, E_{3}=55 \mathrm{MeV}, \gamma_{0}=0.01 \mathrm{~m}^{-1}$, $n=2$. The shaded band indicates the statistical error per $0.02 \mathrm{MeV}$ bin. Right: in the shaded regions, JUNO can distinguish the decoherence scenario from standard oscillations at more than $3 \sigma\left(\Delta \chi^{2}=9\right)$. The red curves show the $90 \%$ C.L. allowed regions from the combined analysis of LSND, KARMEN, Daya Bay and RENO, with the cross indicating the best fit point (same as in figure 3).

exposure of $20 \mathrm{kt}$ detector mass $\times 36 \mathrm{GW}$ reactor power $\times 6 \mathrm{yr}$ exposure $(4320 \mathrm{kt} \mathrm{GW} \mathrm{yr}$ in total) we obtain $10^{5}$ events. The energy resolution is assumed to be $3 \% \sqrt{1 \mathrm{MeV} / E_{\nu}}$. We perform a $\chi^{2}$ analysis using 350 bins for the energy spectrum. Several systematic uncertainties are included as well as the smearing induced by the baseline distribution of 12 relevant reactor cores. Further details of our analysis can be found in [56].

In figure 4 (left) we compare the expected spectra for standard oscillations (red solid) to the decoherence scenario with parameters close to the best fit point (blue dashed). We clearly observe that the $\theta_{13}$-induced modulation of the spectrum (second line in eq. (4.1)) becomes suppressed in the case of decoherence for neutrino energies above $4 \mathrm{MeV}$. Thanks to the huge number of events this difference is highly significant: the $\Delta \chi^{2}$ between those two curves is 33 , which means that the no-decoherence hypothesis would be excluded at more than $5 \sigma$ (1 DOF, i.e., for fixed parameters). The right panel of figure 4 shows the regions in the $\left(E_{1}, E_{3}\right)$ plane, where JUNO will be able to distinguish the decoherence scenario from standard oscillations at more than $3 \sigma\left(\Delta \chi^{2}=9\right)$. We observe that for values of $E_{1}, E_{3} \gtrsim 30 \mathrm{MeV}$, JUNO loses sensitivity, since the decoherence effects will be shifted to higher energies and the reactor neutrino spectrum would be very little modified. We note however, that for such large values of $E_{i}$, decoherence effects may show up in long-baseline or atmospheric neutrino experiments.

Hence, if JUNO does not find any deviation of the energy spectrum from standard oscillations, our scenario would be highly constrained. A dedicated investigation of future data from reactor, long-baseline, and atmospheric neutrino experiments would be required to determine whether an allowed region survives or not. In our analysis we have fixed the exponent in eq. (2.8) to $n=2$. If JUNO would obain results consistent with standard oscillations, one might also test values $n>2$. Increasing $n$, the decoherence for $E<$ 
$E_{1}, E_{3}$ becomes more strongly suppressed which in turn leads to a faster weakening of the sensitivity of JUNO to the parameter range allowed by LSND. Such investigations are beyond the scope of the present work. Note also that we have fixed $\gamma_{0}=0.01 \mathrm{~m}^{-1}$. Smaller values of $\gamma_{0}$ would not allow to fit LSND, as discussed in the previous section. For larger values of $\gamma_{0}$ decoherence effects will become larger at JUNO, increasing the sensitivity.

Finally let us mention that the scenario presented here predicts also large modifications for supernova neutrinos, since the energy range of supernova neutrinos (tens of $\mathrm{MeV}$ ) is close to the LSND energy range, where decoherence effects are important. A discussion of decoherence effects for supernova neutrinos can be found in [10].

\section{Conclusions}

We have revisited the idea of quantum decoherence as a solution to the LSND anomaly proposed in [10] taking into account the recent results from the Daya Bay and RENO reactor experiments. We assume an exponential dependence of the decoherence parameters on neutrino energy as shown in eq. (2.7). For a suitable choice of parameters the decoherence effects can become suppressed for neutrino energies both below and above LSND energies, restricting deviations from the standard three-flavour oscillation scenario to the $20-50 \mathrm{MeV}$ energy range. In this way neither standard oscillations of $\mathrm{MeV}$ neutrinos from the Sun and from reactors are modified, nor the results for neutrinos with energies greater than $200 \mathrm{MeV}$ are affected, as relevant for short and long baseline accelerator experiments and atmospheric neutrinos. Moreover the scenario becomes free from the famous appearancedisappearance tension that plagues the $3+1$ sterile neutrino solution for the LSND anomaly. We have studied the parameter space in which the LSND anomaly can be explained and constraints from various reactor and short baseline neutrino experiments can be avoided. Results are shown in figure 3. We have found a remarkable agreement between KARMEN and LSND data within this scenario. The decoherence solution to LSND predicts no effect in MiniBooNE and is hence consistent with the MiniBooNE null-result for $E_{\nu}>475 \mathrm{MeV}$. However, one should seek another resolution for the low energy excess observed in MiniBooNE between 200 and $475 \mathrm{MeV}$ as well as for the reactor and Gallium anomalies.

The scenario predicts results consistent with standard three-flavour oscillations for most of the upcoming long and short-baseline neutrino oscillation experiments. However, reactor experiments at baselines of around $50 \mathrm{~km}$ such as the JUNO or RENO-50 projects will provide a crucial test of the scenario for large part of the parameter space.

\section{Acknowledgments}

The authors acknowledge partial support from the European Union FP7 ITN INVISIBLES (Marie Curie Actions, PITN- GA-2011- 289442). P.B. is grateful to the Oskar Klein Centre and the CoPS group at Stockholm University for kind hospitality. 
Open Access. This article is distributed under the terms of the Creative Commons Attribution License (CC-BY 4.0), which permits any use, distribution and reproduction in any medium, provided the original author(s) and source are credited.

\section{References}

[1] LSND collaboration, A. Aguilar-Arevalo et al., Evidence for neutrino oscillations from the observation of anti-neutrino(electron) appearance in a anti-neutrino(muon) beam, Phys. Rev. D 64 (2001) 112007 [hep-ex/0104049] [INSPIRE].

[2] J. Kopp, M. Maltoni and T. Schwetz, Are there sterile neutrinos at the eV scale?, Phys. Rev. Lett. 107 (2011) 091801 [arXiv: 1103.4570] [INSPIRE].

[3] J. Kopp, P.A.N. Machado, M. Maltoni and T. Schwetz, Sterile Neutrino Oscillations: The Global Picture, JHEP 05 (2013) 050 [arXiv: 1303.3011] [INSPIRE].

[4] C. Giunti, M. Laveder, Y.F. Li and H.W. Long, Pragmatic View of Short-Baseline Neutrino Oscillations, Phys. Rev. D 88 (2013) 073008 [arXiv:1308.5288] [InSPIRE].

[5] Planck collaboration, P.A.R. Ade et al., Planck 2015 results. XIII. Cosmological parameters, arXiv:1502.01589 [INSPIRE].

[6] J. Bergström, M.C. Gonzalez-Garcia, V. Niro and J. Salvado, Statistical tests of sterile neutrinos using cosmology and short-baseline data, JHEP 10 (2014) 104 [arXiv:1407.3806] [INSPIRE].

[7] S. Gariazzo, C. Giunti and M. Laveder, Light Sterile Neutrinos in Cosmology and Short-Baseline Oscillation Experiments, JHEP 11 (2013) 211 [arXiv:1309.3192] [INSPIRE].

[8] G. Barenboim and N.E. Mavromatos, CPT violating decoherence and LSND: A possible window to Planck scale physics, JHEP 01 (2005) 034 [hep-ph/0404014] [INSPIRE].

[9] G. Barenboim, N.E. Mavromatos, S. Sarkar and A. Waldron-Lauda, Quantum decoherence and neutrino data, Nucl. Phys. B 758 (2006) 90 [hep-ph/0603028] [INSPIRE].

[10] Y. Farzan, T. Schwetz and A.Y. Smirnov, Reconciling results of LSND, MiniBooNE and other experiments with soft decoherence, JHEP 07 (2008) 067 [arXiv: 0805.2098] [INSPIRE].

[11] J.R. Ellis, J.S. Hagelin, D.V. Nanopoulos and M. Srednicki, Search for Violations of Quantum Mechanics, Nucl. Phys. B 241 (1984) 381 [InSPIRE].

[12] S.B. Giddings and A. Strominger, Loss of Incoherence and Determination of Coupling Constants in Quantum Gravity, Nucl. Phys. B 307 (1988) 854 [INSPIRE].

[13] N.E. Mavromatos and S. Sarkar, Probing Models of Quantum Decoherence in Particle Physics and Cosmology, hep-ph/0612193 [INSPIRE].

[14] NuTeV collaboration, S. Avvakumov et al., A search for $\nu_{\mu} \rightarrow \nu_{e}$ and $\bar{\nu}_{m} u \rightarrow \bar{\nu}_{e}$ oscillations at NuTeV, Phys. Rev. Lett. 89 (2002) 011804 [hep-ex/0203018] [INSPIRE].

[15] Daya Bay collaboration, F.P. An et al., Spectral measurement of electron antineutrino oscillation amplitude and frequency at Daya Bay, Phys. Rev. Lett. 112 (2014) 061801 [arXiv: 1310.6732] [INSPIRE].

[16] DAYA BAy collaboration, F.P. An et al., Improved Measurement of Electron Antineutrino Disappearance at Daya Bay, Chin. Phys. C 37 (2013) 011001 [arXiv:1210.6327] [InSPIRE]. 
[17] RENO collaboration, J.K. Ahn et al., Observation of Reactor Electron Antineutrino Disappearance in the RENO Experiment, Phys. Rev. Lett. 108 (2012) 191802 [arXiv: 1204.0626] [INSPIRE].

[18] S.-B. Kim, New results from RENO and prospects with RENO-50, arXiv:1412.2199 [INSPIRE].

[19] Double CHOOZ collaboration, Y. Abe et al., Improved measurements of the neutrino mixing angle $\theta_{13}$ with the Double CHOOZ detector, JHEP 10 (2014) 086 [Erratum ibid. 1502 (2015) 074] [arXiv: 1406.7763] [INSPIRE].

[20] G. Lindblad, On the Generators of Quantum Dynamical Semigroups, Commun. Math. Phys. 48 (1976) 119 [INSPIRE].

[21] T. Banks, L. Susskind and M.E. Peskin, Difficulties for the Evolution of Pure States Into Mixed States, Nucl. Phys. B 244 (1984) 125 [inSPIRE].

[22] Z. Maki, M. Nakagawa and S. Sakata, Remarks on the unified model of elementary particles, Prog. Theor. Phys. 28 (1962) 870 [inSPIRE].

[23] B. Pontecorvo, Neutrino Experiments and the Problem of Conservation of Leptonic Charge, Sov. Phys. JETP 26 (1968) 984 [inSPIRE].

[24] MINOS collaboration, P. Adamson et al., Combined analysis of $\nu_{\mu}$ disappearance and $\nu_{\mu} \rightarrow \nu_{e}$ appearance in MINOS using accelerator and atmospheric neutrinos, Phys. Rev. Lett. 112 (2014) 191801 [arXiv: 1403.0867] [INSPIRE].

[25] T2K collaboration, K. Abe et al., Observation of Electron Neutrino Appearance in a Muon Neutrino Beam, Phys. Rev. Lett. 112 (2014) 061802 [arXiv:1311.4750] [INSPIRE].

[26] T2K collaboration, K. Abe et al., Precise Measurement of the Neutrino Mixing Parameter $\theta_{23}$ from Muon Neutrino Disappearance in an Off-Axis Beam, Phys. Rev. Lett. 112 (2014) 181801 [arXiv: 1403.1532] [INSPIRE].

[27] T2K collaboration, K. Abe et al., Measurements of neutrino oscillation in appearance and disappearance channels by the T2K experiment with 6.6E20 protons on target, arXiv: 1502.01550 [INSPIRE].

[28] SuPER-KamiokAnde collaboration, Y. Ashie et al., Evidence for an oscillatory signature in atmospheric neutrino oscillation, Phys. Rev. Lett. 93 (2004) 101801 [hep-ex/0404034] [INSPIRE].

[29] Super-Kamiokande collaboration, R. Wendell et al., Atmospheric neutrino oscillation analysis with sub-leading effects in Super-Kamiokande I, II and III, Phys. Rev. D 81 (2010) 092004 [arXiv: 1002.3471] [INSPIRE].

[30] MiniBooNE collaboration, A.A. Aguilar-Arevalo et al., A search for electron neutrino appearance at the $\Delta m^{2} \sim 1 e V^{2}$ scale, Phys. Rev. Lett. 98 (2007) 231801 [arXiv:0704.1500] [INSPIRE].

[31] MiniBoonE collaboration, A.A. Aguilar-Arevalo et al., Improved Search for $\bar{\nu}_{\mu} \rightarrow \bar{\nu}_{e}$ Oscillations in the MiniBooNE Experiment, Phys. Rev. Lett. 110 (2013) 161801 [arXiv: 1207.4809] [INSPIRE].

[32] SciBoonE and MiniBoonE collaborations, K.B.M. Mahn et al., Dual baseline search for muon neutrino disappearance at $0.5 \mathrm{eV}^{2}<\Delta m^{2}<40 \mathrm{eV}^{2}$, Phys. Rev. D 85 (2012) 032007 [arXiv:1106.5685] [INSPIRE]. 
[33] F. Dydak et al., A Search for $\nu_{\mu}$ Oscillations in the $\Delta m^{2}$ Range $0.3-90 \mathrm{eV}^{2}$, Phys. Lett. B 134 (1984) 281 [INSPIRE].

[34] NOMAD collaboration, P. Astier et al., Search for $\nu_{\mu} \rightarrow \nu_{e}$ oscillations in the NOMAD experiment, Phys. Lett. B 570 (2003) 19 [hep-ex/0306037] [INSPIRE].

[35] KamLAND collaboration, T. Araki et al., Measurement of neutrino oscillation with KamLAND: Evidence of spectral distortion, Phys. Rev. Lett. 94 (2005) 081801 [hep-ex/0406035] [INSPIRE].

[36] KARMEN collaboration, B. Armbruster et al., Upper limits for neutrino oscillations $\bar{\nu}_{\mu} \rightarrow \bar{\nu}_{e}$ from muon decay at rest, Phys. Rev. D 65 (2002) 112001 [hep-ex/0203021] [INSPIRE].

[37] M.C. Gonzalez-Garcia, M. Maltoni and T. Schwetz, Updated fit to three neutrino mixing: status of leptonic CP-violation, JHEP 11 (2014) 052 [arXiv:1409.5439] [INSPIRE].

[38] M. Maltoni, T. Schwetz, M.A. Tortola and J.W.F. Valle, Ruling out four neutrino oscillation interpretations of the LSND anomaly?, Nucl. Phys. B 643 (2002) 321 [hep-ph/0207157] [INSPIRE].

[39] M. Maltoni and T. Schwetz, Testing the statistical compatibility of independent data sets, Phys. Rev. D 68 (2003) 033020 [hep-ph/0304176] [INSPIRE].

[40] MiniBoonE collaboration, A.A. Aguilar-Arevalo et al., Unexplained Excess of Electron-Like Events From a 1-GeV Neutrino Beam, Phys. Rev. Lett. 102 (2009) 101802 [arXiv: 0812.2243] [INSPIRE].

[41] T. Mueller et al., Improved Predictions of Reactor Antineutrino Spectra, Phys. Rev. C 83 (2011) 054615 [arXiv:1101.2663] [INSPIRE].

[42] P. Huber, On the determination of anti-neutrino spectra from nuclear reactors, Phys. Rev. C 84 (2011) 024617 [Erratum ibid. C 85 (2012) 029901] [arXiv: 1106.0687] [INSPIRE].

[43] G. Mention et al., The Reactor Antineutrino Anomaly, Phys. Rev. D 83 (2011) 073006 [arXiv:1101.2755] [INSPIRE].

[44] C. Giunti and M. Laveder, Statistical Significance of the Gallium Anomaly, Phys. Rev. C 83 (2011) 065504 [arXiv: 1006.3244] [INSPIRE].

[45] NOvA collaboration, R.B. Patterson, The NOvA Experiment: Status and Outlook, Nucl. Phys. Proc. Suppl. 235-236 (2013) 151 [arXiv:1209.0716] [InSPIRE].

[46] LBNE collaboration, C. Adams et al., The Long-Baseline Neutrino Experiment: Exploring Fundamental Symmetries of the Universe, arXiv:1307.7335 [INSPIRE].

[47] The Long Baseline Neutrino Facillity (LBNF), https://web.fnal.gov/project/LBNF/SitePages/Home.aspx.

[48] NUSTORM collaboration, D. Adey et al., Light sterile neutrino sensitivity at the nuSTORM facility, Phys. Rev. D 89 (2014) 071301 [arXiv: 1402.5250] [INSPIRE].

[49] MicroBoonE, LAr1-ND and ICARUS-WA104 collaborations, M. Antonello et al., A Proposal for a Three Detector Short-Baseline Neutrino Oscillation Program in the Fermilab Booster Neutrino Beam, arXiv:1503.01520 [INSPIRE].

[50] A. Anokhina et al., Prospects for the measurement of muon-neutrino disappearance at the FNAL-Booster, arXiv:1404.2521 [INSPIRE]. 
[51] ESSnuSB collaboration, E. Baussan et al., A very intense neutrino super beam experiment for leptonic CP-violation discovery based on the European spallation source linac, Nucl. Phys. B 885 (2014) 127 [arXiv: 1309.7022] [INSPIRE].

[52] M. Blennow, P. Coloma and E. Fernandez-Martinez, Searching for sterile neutrinos at the ESSLSB, JHEP 12 (2014) 120 [arXiv:1407.1317] [INSPIRE].

[53] OscSNS collaboration, M. Elnimr et al., The OscSNS White Paper, arXiv:1307.7097 [INSPIRE].

[54] JUNO collaboration, M. He, Jiangmen Underground Neutrino Observatory, arXiv:1412.4195 [INSPIRE].

[55] Y.-F. Li, J. Cao, Y. Wang and L. Zhan, Unambiguous Determination of the Neutrino Mass Hierarchy Using Reactor Neutrinos, Phys. Rev. D 88 (2013) 013008 [arXiv:1303.6733] [INSPIRE].

[56] M. Blennow, P. Coloma, P. Huber and T. Schwetz, Quantifying the sensitivity of oscillation experiments to the neutrino mass ordering, JHEP 03 (2014) 028 [arXiv:1311.1822] [INSPIRE]. 